POS $\quad$ PROCEEDINGS

\title{
Constraining the dark matter density in galaxy clusters
}

\author{
S. I. Loubser* \\ Centre for Space Research, North-West University, Potchefstroom 2520, South Africa \\ E-mail: ilani.loubser@nwu.ac.za
}

M. Donahue

Department of Physics \& Astronomy, Michigan State University, East Lansing, MI 48824-2320

E-mail: donahue@pa.msu.edu

\section{Voit}

Department of Physics \& Astronomy, Michigan State University, East Lansing, MI 48824-2320

E-mail: voitepa.msu.edu

\begin{abstract}
We illustrate the potential of using stellar velocity dispersion profiles of brightest cluster galaxies (BCGs), in combination with X-ray and weak-lensing derived cluster mass profiles, to constrain the matter distribution from the very centre $(1 \mathrm{kpc})$ to the outskirts $(1000 \mathrm{kpc})$ of galaxy clusters. We have obtained long-slit spectroscopy of a small number of BCGs in CLASH (Cluster Lensing and Supernova Survey with Hubble) clusters at the Southern African Large Telescope (SALT) with the primary goal of measuring their stellar kinematics. The new data extends the observed total mass profile of the cluster over another order of magnitude in radius, which places a much stronger constraint on the dark matter distribution, thereby leveraging the enormous investment in mass-profile measurements at larger radii in these clusters. Here, we discuss the observations and apply the methodology to one of the clusters, RXJ2129.7+0005 $(z \sim 0.23)$, to find results consistent with a mass profile slope that becomes shallower than the typical Navarro-Frenk-White (NFW) profile when dynamical data in the very innermost region of the cluster is also included in its mass profile.
\end{abstract}

SALT Science Conference 2015

1-5 June 2015

Stellenbosch Institute of Advanced Study, South Africa

\footnotetext{
* Speaker.
} 


\section{Introduction}

Despite being a fundamental parameter required to test galaxy formation models (White \& Rees 1978, [16]), the measurement of cluster masses with accuracy on any scale is notoriously difficult. Gravitational lensing offers a particularly promising probe of the total mass profile because of the lack of sensitivity to cluster physics (Kaiser \& Squires 1993, [5]). Projected mass maps of the inner regions of clusters constrained by strongly lensed features of known redshift have been compared to Cold Dark Matter (CDM) model predictions for some time (Tyson, Kochanski \& Dell'Antonio 1993, [13]). Strong and weak lensing provide exquisite mass measurements that are independent of the dynamical state from $30 \mathrm{kpc}$ from the cluster centre to the outskirts. However, gravitational lensing alone cannot separate the baryonic (luminous) and non-baryonic (dark) mass components.

Given the observational evidence that BCGs are gas poor, and often lie at the bottom of the cluster potential in regular, non-interacting systems, the dynamics of the stellar component offers a valuable route to resolving this problem. In practice, the stellar kinematics of the BCG provides an additional measure of the total mass at small radii since the stellar velocity dispersion probes the depth of the gravitational potential well in which the stars are sitting. Measuring the BCG stellar velocity dispersion profile allows us to dynamically probe the central mass distribution in a region where X-ray measurements are often distorted by AGN outflows, and weak lensing and SunyaevZel'dovich (SZ) measurements lack the resolution required to obtain a good constraint on the slope of the total mass distribution. Therefore, combining the different mass probes is necessary to derive comprehensive constraints on the total mass distribution in clusters, and the distribution of the dark matter component.

Simulations of increasing resolution and volume confirm the essential findings of the Navarro, Frenk \& White (1996, hereafter NFW [9]) description of the dark matter profile in clusters (Gao et al. 2012, [4]), although there has been some controversy over the refinement of the detailed profile shape. The form of the dark matter profile remains a robust and testable prediction of the CDM paradigm.

We combine new SALT spectroscopic measurements of the stellar velocity dispersion profile of the BCG in CLASH cluster RXJ2129.7+0005 with the weak lensing (Umetsu et al. 2014 [14], Merten et al. 2014 [8]) and X-ray (Donahue et al. 2014, [3]) derived mass profiles to constrain the distribution of total mass over $1000 \mathrm{kpc}$ of radius in the cluster. The inclusion of the long-slit spectra extends the observed mass profile to include the centre of the cluster, where baryonic matter becomes increasingly important, and it places a much stronger constraint on the dark matter component distribution, leveraging the enormous investment in mass-profile measurements at larger radii. Throughout this study we adopt a $\Lambda \mathrm{CDM}$ cosmology with $\Omega_{m}=0.3, \Omega_{\Lambda}=0.7$, and $H_{0}=70$ $\mathrm{km} \mathrm{s}^{-1} \mathrm{Mpc}^{-1}$.

\section{Data}

The CLASH survey consists of 25 massive intermediate redshift galaxy clusters observed with the Hubble Space Telescope (HST) to accurately constrain their mass distributions. The clusters are solely X-ray selected, massive $(\mathrm{kT}>5 \mathrm{keV})$ and, in most cases, dynamically relaxed. BCGs in the 


\begin{tabular}{llr}
$\alpha_{J 2000}$ & & $21: 29: 40$ \\
$\delta_{J 2000}$ & & $+00: 05: 21$ \\
$z$ & $(\mathrm{kpc} / \mathrm{h})$ & 0.234 \\
$R_{\text {off }}$ & 6.3 \\
X-ray derived central entropy $K_{0}$ & $\left(\mathrm{keV} \mathrm{cm}{ }^{2}\right)$ & 21.1 \\
BCG exposure time on SALT & (seconds) & 18000 \\
BCG major axis and slit P.A. & (degrees) & 61 \\
\hline
\end{tabular}

Table 1: RXJ2129.7+0005 properties, and the details of the long-slit observations. $R_{o f f}$ is the projected offset between the centre of the BCG and the X-ray peak.

CLASH survey have not yet been followed up with detailed optical spectroscopy and most CLASH optical auxiliary data consists of photometry and spectroscopy of the surrounding galaxies. Here, we focus on the total mass distribution in one CLASH cluster, RXJ2129+0005 (shown in Table 1, to demonstrate the potential of the method.

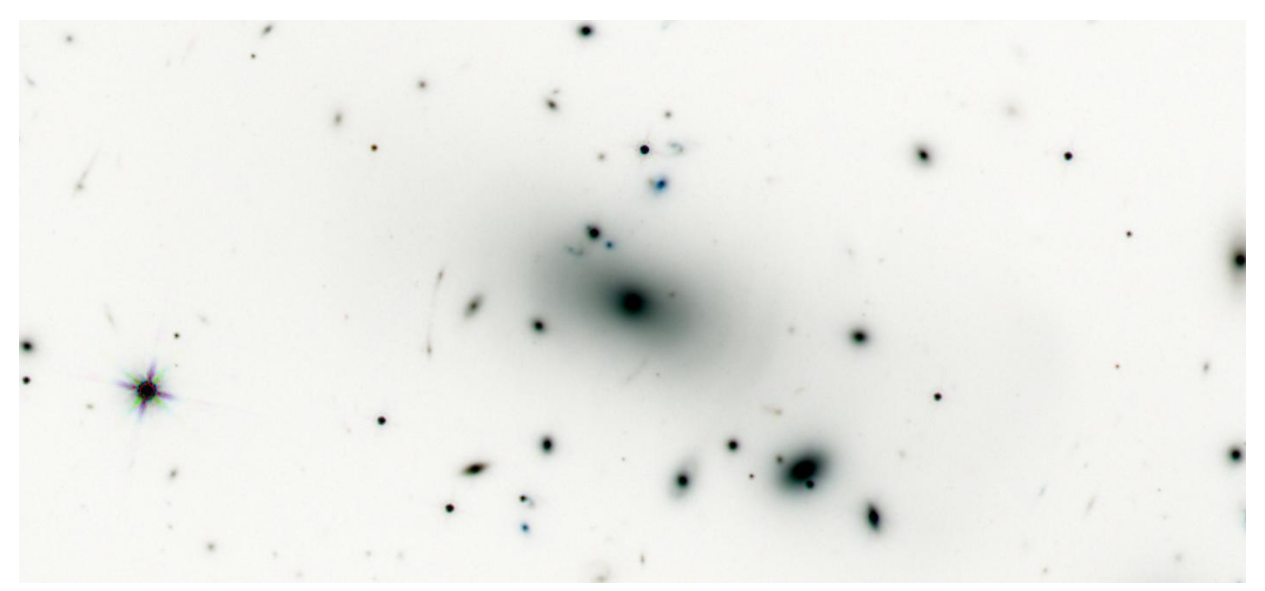

Figure 1: RXJ2129.7+0005 (from the Hubble Legacy archive, http://hla.stsci.edu).

\subsection{Optical spectroscopy and data reduction}

The data for RXJ2129.7+0005 were obtained with the Robert Stobie Spectrograph (RSS) on the SALT telescope between April 2013 and September 2013 (2013-1-RSA_OTH-011; PI: I Loubser). The PG0900 grating, with a 1.5" slit placed on the galaxy's major axis, and set at 35.5 degrees delivered the required wavelength range to observe $\sim 20$ optical absorption lines at $z=0.23$ for an accurate measurement of the stellar velocity dispersion as a function of galactocentric radius. A total of 14 tracks were observed in seeing conditions between 1 and 2". In addition, the necessary flat-field and arc frames were also observed at regular intervals, as well as spectrophotometric standard stars for flux calibration.

The basic reductions were done using the PySALT: SALT science pipeline ${ }^{1}$ (Crawford et al.

\footnotetext{
${ }^{1}$ http://pysalt.salt.ac.za/
} 
2010, [2]), whilst further reductions were done in $\operatorname{IRAF}^{2}$. Frames were mosaiced, overscan regions were trimmed, and flat-field frames were used to correct for differences in sensitivity both between detector pixels and across the field. The majority of the cosmic rays were rejected in the individual frames using the LACosmic routine (van Dokkum 2001, [15]) before subtraction of the background sky continuum and emission lines. The spectra were carefully wavelength calibrated using the arc lamp spectra. A spectrophotometric standard star was used to correct the measured counts for the combined transmission of the instrument, telescope and atmosphere as a function of wavelength. We observed and reduced the standard star with the same instrument configuration as the corresponding scientific data. A single central spectrum, consisting of $\sim 10$ spatial rows from the maximum luminosity peak, was extracted and used to convert the measured counts from the galaxy spectra into fluxes with erg $\mathrm{cm}^{-2} \mathrm{~s}^{-1} \AA^{-1}$ units. The individual science frames were then combined to produce a one 2-D spectrum of the BCG in RXJ2129.7+0005.

The galaxy and propagated error spectra were binned in the spatial direction to ensure a minimum signal-to-noise ratio $(\mathrm{S} / \mathrm{N})$ of 20 per $\AA$ in the $\mathrm{H} \beta$ region of the spectrum for kinematics measurements. A $\mathrm{S} / \mathrm{N}$ ratio of 20 per bin was chosen to resolve the optimal number of possible measurement points with acceptable errors $(<10 \%)$ on the measurements. As a result, the spatial bins become wider with increasing radius from the centre of the galaxy.

\subsection{Kinematic measurements}

We used the PPXF routine ${ }^{3}$ by Cappellari \& Emsellem (2004, [1]), and the MILES stellar library (Sánchez-Blázquez et al. 2006, [11]) to derive the stellar kinematics. We increased the S/N ratios of the spatial bins by combining both spatial sides of the galaxy (at the same distance from the centre) before the kinematics are measured, making it possible to reach 10 arcsec from the centre of the galaxy and having at least four bins. This assumed symmetry in the major axis kinematics of the BCG is justified given the agreement between independent measurements (see e.g. Newman et al. 2013, [10]), and because the dynamical models used to interpret BCG kinematics assume spherical symmetry. The assumption of spherical symmetry in the dynamical models is mainly to prevent the introduction of additional degrees of freedom which would make the system underdetermined, although axisymmetrical and triaxial models are being developed.

\section{Mass distribution profile results}

This section presents the mass profile derived for RXJ2129.7+0005 from all the available data sets. The Joint Analysis of Cluster Observations (JACO) code is used to combine X-ray, SZ, and weak lensing data to simultaneously constrain the shapes of the mass profiles in clusters (Mahdavi et al. 2007, [7]). The cluster mass profile is presented (in Figure 2) as circular-velocity plot which is virtually independent of cosmology and therefore allows mass profiles derived assuming different cosmologies to be plotted simultaneously. It serves as an extension of Figure 4 in Donahue et al. (2014, [3]), and shows the change in the profile slope when the centre of the cluster is included in the fit.

\footnotetext{
${ }^{2}$ IRAF is distributed by the National Optical Astronomy Observatories, which are operated by the Association of Universities for Research in Astronomy Inc., under cooperative agreement with the National Science Foundation.

${ }^{3}$ We make use of the PPXF code which can be retrieved at http:/www.leidenuniv.nl/sauron/.
} 
Based on the agreement between the mass profiles derived from lensing and X-ray observations, we can also infer that the cluster is not strongly elongated along the line-of-sight and the intracluster medium is close to equilibrium.

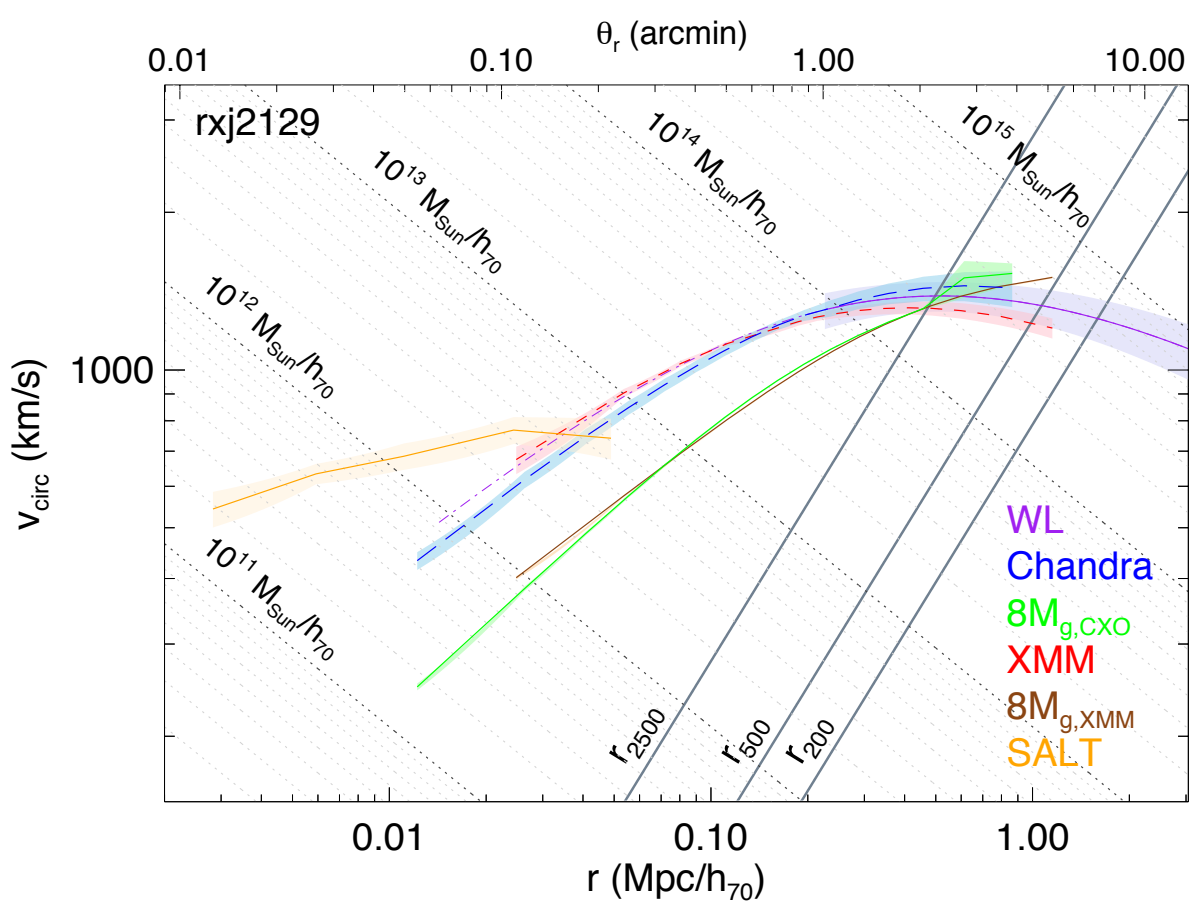

Figure 2: RXJ2129.7+0005 mass profile. The plot shows that the hydrostatic equilibrium mass profile derived from Chandra observations (blue line, Donahue et al. 2014, [3]) closely resembles that determined from lensing (purple line, Umetsu et al. 2014, [14]). The red line shows the mass profile derived from JACO fitting using XMM data, and the hatched regions shows 68 per cent confidence intervals. The bottom axis shows the radial distance (in $h_{70}^{-1} \mathrm{Mpc}$ ) from the cluster centre which is uncertain by less than 1 per cent below $z<0.5$. Also shown are gas mass profiles multiplied by a factor of 8 (the ratio of gas mass to total mass is 1:8 at $r_{2500}$ ) from the parametric analysis of JACO from Chandra and XMM data. The yellow line shows the mass profile in the very centre of the cluster as derived from SALT observations. The light diagonal lines show enclosed mass, and the dark diagonal lines shows the loci of the spherical overdensity radii $r_{2500}, r_{500}$ and $r_{200}$.

\section{Conclusions}

CLASH has been testing the $\Lambda$ CDM model by measuring radial cluster mass profiles derived from gravitational lensing and comparing them with simulated profiles of $\Lambda \mathrm{CDM}$ clusters. They found excellent agreement, and that the clusters are generally well described by an NFW profile (Umetsu et al. 2014, [14]). The main result of this paper is that for RXJ2129.7+0005, the mass profile slope becomes shallower when data describing dynamics in the very centre of the cluster 
(the BCG stellar velocity dispersion) is included in the fit. This is also consistent with the results by Newman et al. (2013, [10]) for their similar study of seven clusters $(0.2<z<0.3)$. The effects on baryons become increasingly important on smaller scales, and dark matter is gravitationally important on larger scales. Therefore, only the full observed structure of observed haloes may inform us about the interplay between baryons and dark matter, which relates to the formation and assembly histories of clusters and galaxies.

Laporte \& White (2014, [6]) showed, using $\Lambda$ CDM simulations, that multiple dissipation-less mergers can cause the cluster mass profiles to become slightly shallower than the predicted NFW, and that these differences are found at the radii where the influence of the black hole is significant $\left(r / r_{200}<0.015\right)$, similar to the half-light radius of the BCG. Schaller et al. (2014, [12]), on the other hand, found that the mass distributions in their simulations is very well described by an NFW profile, and they argue that the inconsistency between the observed results and their profiles are at least partly due to the assumption of isotropy in interpreting the observational data. The analysis needs to be extended to more clusters to solve these discrepancies, and to answer the important question of whether cluster mass profiles are universal across all halo masses.

\section{Acknowledgments}

SIL is financially supported by the South African National Research Foundation. All of the observations reported in this paper were obtained with the Southern African Large Telescope (SALT) under program 2013-1-RSA_OTH-011 (PI: Ilani Loubser).

\section{References}

[1] Cappellari, M., \& Emsellem, E., 2004, PASP, 116, 138

[2] Crawford, S. M., Still, M., Schellart, P., et al., 2010, in SPIE Conference Series, Vol. 7737, 25

[3] Donahue, M., Voit, G. M., Mahdavi, A., et al., 2014, ApJ, 794, 136

[4] Gao, L., Navarro, J. F., Frenk, C. S., et al., 2012, MNRAS, 425, 2169

[5] Kaiser, N., \& Squires, G., 1993, ApJ, 404, 441

[6] Laporte, C. F. P., \& White, S. D. M., 2015, MNRAS, 451, 1177

[7] Mahdavi, A., Hoekstra, H., Babul, A., et al., 2007, ApJ, 664, 162

[8] Merten, J., Meneghetti, M., Postman, M., et al., 2015, ApJ, 806, 4

[9] Navarro, J. F., Frenk, C. S., \& White, S. D. M., 1996, ApJ, 462, 563

[10] Newman, A. B., Treu, T., Ellis, R. S., \& Sand, D. J., 2013, ApJ, 765, 25

[11] Sánchez-Blázquez, P., Peletier, R. F., Jimenez-Vicente, J., et al., 2006, MNRAS, 371, 703

[12] Schaller, M., Frenk, C. S., Bower, R. G., et al., 2015, MNRAS, 452, 343

[13] Tyson, J. A., Kochanski, G. P., \& Dell'Antonio, I. P., 1998, ApJL, 498, L107

[14] Umetsu, K., Medezinski, E., Nonino, M., et al., 2014, ApJ, 795, 163

[15] van Dokkum, P. G., 2001, PASP, 113, 1420

[16] White, S. D. M., \& Rees, M. J., 1978, MNRAS, 183, 341 